\title{
SOP Endotracheales Absaugen - unter Beteiligung der Hygiene
}

Jens Jakisch, Renate Rettkowski

\begin{abstract}
Das endotracheale Absaugen ist eine Routinetätigkeit auf Intensivstationen. Die natürlichen Abwehrmechanismen der Atemwege sind durch den Tubus weitestgehend ausgeschaltet. Neben der korrekten Technik ist deshalb die Beachtung der hygienischen Vorgaben zur Infektionsvermeidung entscheidend. Daher ist es sinnvoll, neben den Experten von Station auch Fachleute der Hygiene in die SOPErstellung einzubeziehen.
\end{abstract}

\section{Einleitung}

\section{Hintergrund}

Die von den Fachabteilungen geschrieben SOPs bestechen in der Regel durch ihre praxisnahen Beschreibungen der Arbeitsabläufe. Hygieneaspekte werden aber oft nur unzureichend be(tr)achtet. Die von den Hygieneabteilungen verfassten SOPs lassen den Anwender aufgrund fehlender Kenntnisse der tatsächlichen Arbeitsabläufe - z. B. in der Intensivmedizin - oft über die Umsetzung in der Praxis im Unklaren.

Wir wollen anhand dieser SOP zeigen, dass eine adäquate Hygiene praxisnah in die Arbeitsabläufe integriert werden kann und dass eine enge Zusammenarbeit zwischen den Fachabteilungen und der Hygiene

$\begin{array}{ll}\text { ABKÜRZUNGEN } & \\ \text { FiO2 } & =\text { fraction of inspired oxygen } \\ & \text { (Sauerstoffanteil der } \\ & \text { Einatemluft) } \\ & =\text { high frequency oscillation } \\ \text { HFOV } & \text { ventilation (Oszilierende Hoch- } \\ & \text { frequenzbeatmung) } \\ & =\text { Kommission für Krankenhaus- } \\ \text { KRINKO } & \text { hygiene und Infektionspräven- } \\ & \text { tion } \\ \text { MRE } & =\text { Multi-Resistente Erreger } \\ \text { NO-Beatmung } & =\text { Beatmung mit Stickstoff- } \\ & \text { monoxid } \\ \text { PEEP } & =\text { positive endexpiratory } \\ & \text { pressure (Positiver endexspira- } \\ & \text { torischer Druck) } \\ \text { PSA } & =\text { Persönliche Schutzausrüstung } \\ \text { RKI } & =\text { Robert Koch Institut } \\ \text { SOP } & =\text { standard operating procedure } \\ & \text { (Standardarbeitsanweisung) }\end{array}$

gewinnbringend im Sinne des Patientenwohls für alle Seiten ist.

\section{Verfahren/Umfang dieser SOP}

Die vorliegende SOP behandelt das endotracheale Absaugen von beatmeten Intensivpatienten. Das Vorgehen auf Normalstation erfolgt analog.

\section{Erläuterungen}

Hygienische Basismaßnahmen

Hygienische Basismaßnahmen - wie zum Beispiel die hygienische Händedesinfektion vor Manipulationen am Beatmungssystem oder das Vorbereiten auf einer wischdesinfizierten Fläche - werden häufig in SOPs als selbstverständlich vorausgesetzt und deshalb nicht beschrieben. Wenn diese allerdings in einer detaillierten Tätigkeitsbeschreibung fehlen, kann dies dem Anwender suggerieren, dass sie nicht notwendig seien. Hygienemaßnahmen sollten in die Beschreibung des Arbeitsablaufes integriert werden, um die Compliance zur Einhaltung zu erhöhen. Eine konsequente Hände- und Arbeitsplatzhygiene mit Trennung von rein/unrein, korrekter Sterilgutlagerung und der regelmäßigen Desinfektion von häufigen Handkontaktstellen reduziert das Risiko einer Keimverschleppung.

\section{Wie häufig absaugen?}

Das routinemäßige Absaugen ist wegen der möglichen Komplikationen - z. B. Verletzungen - und der Belastung für den Patienten (Schmerzen, Erstickungsgefühl) obsolet! Unnötige Diskonnektionen müssen aus hygienischen und beatmungstechnischen Gesichtspunkten vermieden werden. Jede Diskonnektion birgt die Gefahr der Kontamination des Beatmungssystems. Jeder Absaugvorgang reizt zudem die Schleimhäute der Atemwege und regt die Produktion von respiratorischem Sekret an. 
CHECKLISTE: VORBEREITUNG BEIM GESCHLOSSENEN

ENDOTRACHEALEN ABSAUGEN

- An erster Stelle stehen die Hände- und die Arbeitsflächendesinfektion.

- Vorbereitung der sterilen Flüssigkeit (üblicherweise $\mathrm{NaCl}$ $0,9 \%$ oder Aqua) zur Spülung des geschlossenen Systems [1] nach dem Absaugvorgang unter aseptischen Kautelen. Mehrdosisbehältnisse, z. B. 50 ml- oder 100 ml-Fläschchen, die zur Mehrfachentnahme von Spülflüssigkeit patientennah deponiert werden, sind wegen des fehlenden Konservierungsmittels und nicht gesicherter Sterilität abzulehnen.

- Der Anschluss-Port für die Spülflüssigkeit sollte vor Verwendung mit einem geeigneten Desinfektionsmittel (It. Herstellerangaben) wischdesinfiziert werden, da dieser einen direkten Zugang zum Inneren des Beatmungssystems darstellt und im unmittelbaren, potenziell kontaminierten Patientenumfeld liegt.

- Zur Mobilisation von Atemwegssekret verwendete instillierte Flüssigkeit muss steril sein. Allerdings sollte die Indikation zum Einsatz von Flüssigkeit zur Atemwegssekretmobilisation streng gestellt werden. Neben dem Diskomfort für den Patienten (Gefühl des Ertrinkens) kann es zu einer Dislozierung von Erregern aus dem Biofilm des Tubus oder von Sekret aus den oberen Atemwegen mit Einbringung in die unteren Atemwege kommen.

- Beim geschlossenen Absaugen ohne Exposition zu respiratorischen Sekreten sind weder Mund-Nasenschutz noch Einwegschürze erforderlich.

\section{Merke}

Für das endotracheale Absaugen gilt die Faustregel „So wenig wie möglich, so oft wie nötig!“

\section{Indikationen}

Die Indikation zum Absaugen tritt oft plötzlich und eindrucksvoll auf. Der Patient hustet, läuft rot an, Sekret ist im Tubus zu sehen, das Beatmungsgerät alarmiert und die Vitalwerte erreichen bedenkliche Bereiche. Es ist dennoch wichtig, einen kühlen Kopf zu bewahren, um die wichtigsten Hygieneregeln einzuhalten. Die Vermeidung von Infektionen rechtfertigt hier einen Moment des Diskomforts. In diesen Fällen kann eine Analgetika -/Sedativagabe die nötige Zeit verschaffen.

- Sichtbares Sekret im Tubus/Beatmungssystem, Rasselgeräusche oder ein „Sägezahnmuster“ in der Volumen-Flow-Kurve, gegebenenfalls mit Abfall der Sauerstoffsättigung zeigen eine Absaugindikation an.

- Die Gewinnung von Trachealsekret zu diagnostischen Zwecken sollte nach Möglichkeit bei einem ohnehin indizierten Absaugvorgang erfolgen.

\section{Geschlossenes und offenes endotracheales Absaugen}

Beim endotrachealen Absaugen unterscheidet man zwischen dem offenen und dem geschlossenen Absaugen:

Offenes Absaugen Das Beatmungssystem wird vom Endotrachealtubus diskonnektiert und ein steriler Absaugkatheter wird in den Tubus eingeführt.

Geschlossenes Absaugen Es wird mit einem mehrfach verwendbaren, in das Beatmungsschlauchsystem integrierten Absaugkatheter ohne Diskonnektion abgesaugt.

Laut KRINKO-Empfehlung ist dabei kein Verfahren aus infektiologischer Sicht überlegen [1,2]. Allerdings wird eine geschlossene Absaugung für Patienten mit Tröpfchen- und Luftübertragbaren Infektionskrankheiten (z.B. Tuberkulose, Windpockenpneumonie) sowie MRE-Kolonisation/Infektion der Atemwege ausdrücklich empfohlen. Die Einbringung von Erregern in das Patientenumfeld und eine mögliche Kontamination des Pflegepersonals beim Absaugvorgang sollen so vermieden werden. Beatmungstechnische Indikationen für die Verwendung einer geschlossenen Absaugung wären zum Beispiel ein hoher PEEP (ab $10 \mathrm{~cm}$ $\mathrm{H}_{2} \mathrm{O}$ ), Bauchlagerung des Patienten, NO- oder HFOVBeatmung. Ohne Diskonnektion wird ein PEEP-Verlust und damit das Kollabieren von Alveolen vermieden.

\section{Geschlossenes endotracheales Absaugen}

Vorbereitung Neben der technischen Überprüfung der Absaugeinheit und dem Bereitstellen aller benötigten Materialien gibt es bei der Vorbereitung zum geschlossenen endotrachealen Absaugen wesentliche hygienische Aspekte.

Vor dem Absaugvorgang erfolgt bei Indikation die Präoxygenierung - eine kurzzeitige Erhöhung der $\mathrm{FiO}_{2}$ manuell oder per Bedienfeld - um dem Patienten eine Sauerstoffreserve zu verschaffen.

\begin{abstract}
Merke
Zur Stressreduktion kann eine kurzzeitige Vertiefung der Sedierung oder eine Analgetikagabe, besonders bei instabilen Patienten, z. B. mit frischen CoronarBypässen oder Hirndrucksymptomatik, sinnvoll sein.
\end{abstract}

Durchführung Der Absaugschlauch wird mit der geschlossenen Absaugung verbunden und der Sog mit maximal $200 \mathrm{~mm} \mathrm{Hg}$ angewählt, um Schleimhautverletzungen zu vermeiden. 
- Konventionelle Absaugkatheter werden ohne Sog eingeführt.

- Atraumatische Absaugkatheter werden unter Sog eingeführt. Sie entwickeln durch einen ringförmigen Wulst und seitliche Öffnungen an der Katheterspitze unter Sog ein Luftkissen, das ein Ansaugen an die Trachealwand und damit Schleimhautverletzungen verhindern soll.

Die den Katheter umgebene Schutzhülle muss sauber nachgeführt und darf nicht beschädigt werden, da es sonst zu einer Leckage kommt, die einen Systemwechsel erforderlich macht.

Zur Sekretentfernung aus dem System nach dem Absaugen wird das Absaugventil bei vollständig zurückgezogenem Absaugkatheter betätigt und gleichzeitig über die aufgesetzte Spritze die sterile Spülflüssigkeit zugespritzt, bis kein Sekret mehr im System steht. Anschließend muss die Spritze mit der restlichen Spülflüssigkeit verworfen werden, da sonst eine Keimvermehrung darin droht.

\section{Offenes endotracheales Absaugen}

Vorbereitung Oft ist Eile geboten, da sich der Patient unter akutem Sekretverhalt vital verschlechtert. Der Ablauf muss so strukturiert sein, dass die Händedesinfektion an der (den) entscheidenden Stellen durchgeführt wird, wenn mehrere Tätigkeiten nacheinander am Patienten durchgeführt werden.

\section{Merke}

Die wichtigste hygienische Maßnahme zur Vorbereitung des offenen endotrachealen Absaugens ist die hygienische Händedesinfektion zum richtigen Zeitpunkt im Rahmen der Vorbereitung mit mehreren Arbeitsschritten.

Absaugkatheter Für ein glattes Vorschieben wählt man einen Absaugkatheter mit einem Außendurchmesser halb so groß wie der Innendurchmesser des Endotrachealtubus. (Beispiel: Tubus 8,0 mm Innendurchmesser $=12$ Ch (4,0 mm) Absaugkatheter).

Präoxygenierung Die Präoxygenierung hat bei der offenen endotrachealen Absaugung neben der Bereitstellung einer Sauerstoffreserve noch einen wichtigen hygienischen Aspekt. Bei den meisten Beatmungsgeräten wird die Beatmung bei Erkennung einer Diskonnektion im Präoxygenierungsmodus unterbrochen. Dadurch wird weniger potenziell kontaminiertes Aerosol aus dem Beatmungssystem in die Umgebung abgegeben. Oft bildet sich Kondensat mit patienteneigener pathologischer Kolonisation am tubusnahen Ende des Schlauchsystems. Analog zur geschlossenen Absaugung kann eine Vertiefung der Sedierung oder eine
CHECKLISTE: VORBEREITUNG BEIM OFFENEN

ENDOTRACHEALEN ABSAUGEN

- Flüssigkeit zur Sekretmobilisation (s. o.) oder auch Probenbehälter müssen unter aseptischen Kautelen auf einer wischdesinfizierten (Arbeits-)Fläche zu- bzw. vorbereitet werden.

- Da beim offenen endotrachealen Absaugen mit dem Verspritzen von respiratorischen Sekreten zu rechnen ist, ist das Tragen eines Mund-Nasenschutzes bzw. einer FFP-Maske (z. B. Tuberkulose, Varizellen) und einer Einmalschürze zusätzlich zu Einmalhandschuhen erforderlich, ggf. auch eine Schutzbrille [3].

- Es werden zudem sterile Handschuhe und sterile Absaugkatheter empfohlen [1]. Dabei ist ein steril verpackter Copolymerhandschuh über der Führungshand ausreichend.

- Ein oft vernachlässigter Aspekt ist die korrekte Aufbewahrung der sterilen Handschuhe und Absaugkatheter (Sterilgut) am Bettenplatz.

Analgetikagabe angezeigt sein (siehe auch geschlossenes Absaugen).

Durchführung Die Durchführung der offenen endotrachealen Absaugung lässt auf Grund des Schnelligkeitsgebots und des hygienisch sicheren Handelns wenig Raum für Variationen. Es sollte ein routinierter, einheitlicher Ablauf eingeübt werden, da auch die manuelle Handhabung anspruchsvoll ist. Dabei müssen Tätigkeiten mit der „reinen“ (mit sterilem Einmalhandschuh) und der „unreinen“ Hand getrennt werden. Allein aus dieser Grundüberlegung ergibt sich ein relativ festes Schema. Der gesamte Vorgang sollte nicht länger als 10-15 Sekunden dauern, da eine Oxygenierung des Patienten während des Absaugens nicht stattfindet.

\section{Vorgehen}

1. Nach der Händedesinfektion (Indikation: vor aseptischen Tätigkeiten) und Anlegen der keimarmen Schutzhandschuhe wird die Verpackung des Absaugkatheters am Konusende in Peel-off-Technik nur so weit geöffnet wie es erforderlich ist, um den Absaugkatheter mit dem Absaugschlauch zu verbinden.

2. Danach wird der sterile Einmalhandschuh über den unsterilen Einmalhandschuh der Führungshand angelegt. Die Verpackung kann als saubere Ablagefläche genutzt und unter das patientennahe Beatmungssystem gelegt werden. Es kämen auch andere Möglichkeiten in Frage, aber aus Zeit- und HandlingGründen ist die Nutzung der Handschuhverpackung die praktikabelste (und günstigste) Methode.

3. Es folgt ein manuell anspruchsvoller Schritt: Die Entfernung der Hülle des Absaugkatheters. Dabei wird der Katheter mit der unsterilen Hand am Fingertip langsam aus der Verpackung gezogen, während die sterile Hand ihn in Empfang nimmt. Zudem 


\section{EMPFEHLUNGEN DER KRINKO ZUM ENDOTRACHEALEN} ABSAUGEN

- Unter infektionspräventiven Gesichtspunkten konnte kein Unterschied zwischen offenen und geschlossenen Absaugsystemen gezeigt werden (Kat IA).

- Da ein routinemäßiger täglicher Wechsel keinen Einfluss auf die Inzidenz der VAP hat, empfiehlt es sich, geschlossene Absaugsysteme zu verwenden, die längere Wechselintervalle zulassen, wobei das System mindestens einmal wöchentlich gewechselt werden sollte (Kat II).

- Zur Vermeidung einer zusätzlichen Umgebungskontamination und Exposition des Personals beim Absaugen sind bevorzugt geschlossene Absaugsysteme einzusetzen, wenn die Patienten in den Atemwegen mit multiresistenten Erregern (MRE) kolonisiert sind oder eine Atemwegsinfektion mit MRE aufweisen (Kat II).

- Beim offenen endotrachealen Absaugen sind sterile Katheter und sterile Handschuhe zu verwenden und anschließend an die einmalige Nutzung zu verwerfen. Zur Spülung des vom Patienten bereits diskonnektierten Überleitungsschlauches zum Behältnis für Absaugflüssigkeit ist keimarme Flüssigkeit ausreichend.

- Zur Mobilisierung von Atemwegssekret sind sterile Flüssigkeiten zu verwenden.

- Beim geschlossenen Absaugen muss das gesamte System nach Gebrauch mit steriler Flüssigkeit gespült werden.

DIE 5 INDIKATIONEN ZUR HÄNDEDESINFEKTION NACH WHO MODIFIZIERT AM BEISPIEL DES ENDOTRACHEALEN ABSAUGENS

- Unmittelbar vor Patientenkontakt $\rightarrow$ vor Anlegen der keimarmen Handschuhe; vor einer Lagerung des Patienten

- Vor aseptischen Tätigkeiten $\rightarrow$ vor Aufziehen der sterilen Spülflüssigkeit/sterilen Flüssigkeit zur Sekretmobilisation; vor Kontakt mit dem Beatmungssystem

- Nach Patientenkontakt $\rightarrow$ nach Ablegen der keimarmen Handschuhe

- Nach Kontakt mit kontaminiertem Material/Flüssigkeiten $\rightarrow$ Nach Kontakt mit respiratorischem Sekret

- Nach Kontakt mit der unmittelbaren Patientenumgebung $\rightarrow$ Nach Kontakt mit dem Absaugsystem; nach Kontakt mit dem Beatmungsgerät

muss die Verpackung vorsichtig fixiert werden, damit sich der Katheter aus der Packung lösen lässt. Bei Kontamination muss ein neuer Absaugkatheter verwendet werden. Es empfiehlt sich, den Absaugkatheter beim Auspacken in einer Schlaufe zu fassen, was die Handhabung erleichtert.

4. Nun wird das Beatmungssystem mit der unsterilen Hand vom Tubus diskonnektiert und auf der sauberen Ablagefläche abgelegt. Sofern das Beatmungs- gerät eine aktivierte Präoxygenierungsfunktion hat, sollte die Beatmung jetzt automatisch pausieren. Das Beatmungssystem sollte so abgelegt werden, dass möglichst wenig kontaminiertes Sekret in die Umgebung verblasen wird - mit der Öffnung zur Ablage.

5. Der Absaugkatheter wird zügig mit der sterilen Hand in den Tubus eingeführt, während die unsterile Hand diesen zur Stabilisierung fixiert. Der Absaugkatheter wird bis kurz hinter das Ende des Endotrachealtubus und/oder bis zum Auslösen eines Hustenstoßes vorgeschoben. Ein zu forsches und zu tiefes Einführen des Absaugkatheters bis zu einem Widerstand kann zu Verletzungen der Trachealschleimhaut und damit Eintrittspforten für Infektionen führen.

6. Nach dem Einführen wird der Absaugkatheter langsam unter Sog zurückgezogen und dabei spiralig um den Zeigefinger der sterilen Hand gewickelt, während die unsterile Hand den Tubus fixiert. Durch die langsame spiralige Bewegung soll möglichst viel Sekret entfernt werden.

7. Wenn der Katheter den Tubus verlassen hat, wird der sterile Handschuh über den Katheter gestülpt und beides verworfen. Anschließend wird das Beatmungssystem wieder mit dem Tubus verbunden. Dabei ist darauf zu achten, dass die Konnektionsstellen innen nicht berührt werden.

8. Der Absaugschlauch wird mit keimarmer Flüssigkeit durchgespült und der potenziell kontaminierte Fingertip senkrecht in einer dafür vorgesehenen Halterung aufgehängt, um eine Kontamination der Umgebung zu vermeiden. Die keimarme Flüssigkeit sowie der Absaugschlauch sollten arbeitstägig sowie bei Patientenwechsel ausgetauscht werden. Moderne Sekretauffangbehältnisse sind meist geschlossen und ohne Aufbereitung der Absauganlage austauschbar. Deshalb können sie in der Regel bis zur vollständigen Füllung oder Patientenwechsel am Platz verbleiben. Offene Sekretauffangbehältnisse sollten dagegen täglich gewechselt und aufbereitet werden.

9. Angefallene Abfälle (z. B. Verpackungen) werden entsorgt.

10. Kontaminierte Flächen und Handkontaktstellen müssen wischdesinfiziert werden, um Kreuzkontaminationen mit erregerhaltigen Materialien zu vermeiden. Dies sollte auch immer den Hebel/ Schalter/Drehregler zum Betätigen der Absauganlage beinhalten, da dieser erfahrungsgemäß häufig genutzt wird.

11. Nach Abschluss dieser Maßnahme kann die verwendete PSA abgelegt werden. Dabei ist zu beachten, dass diese von stark nach schwach kontaminiert abzulegen ist, also die keimarmen Schutzhandschuhe vor der Einmalschürze und der optionalen Schutzbrille ausziehen. 
12. Der letzte Arbeitsschritt ist die hygienische Händedesinfektion. Indikation(en) sind „nach Patientenkontakt“ und „nach Kontakt mit kontaminiertem Material“, hier respiratorisches Sekret.

Fazit Von der Vorbereitung über die Durchführung bis hin zur Nachbereitung: Nur das Einbetten der gebotenen hygienischen Maßnahmen in den technischen Ablauf des endotrachealen Absaugens gewährleistet ein Höchstmaß an Sicherheit für den Patienten (und des Personals). So ziemlich jede ärztliche oder pflegerische Intervention hat auch eine hygienisch relevante Dimension. Diese ist für nicht hygienisches Fachpersonal oft nicht sicher abzuschätzen. Deshalb sollte bei der Erstellung von Arbeitsanweisungen/SOPs auch immer hygienisches Fachpersonal hinzugezogen werden. So können sinnvolle Arbeitsabläufe in der Klinik implementiert werden, die helfen, Infektionen und damit Leid und Kosten zu minimieren.

\section{Interessenkonflikt}

Die Autoren geben an, dass kein Interessenkonflikt besteht.

\section{Über die Autoren}

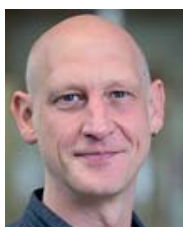

\section{Jens Jakisch}

Jens Jakisch, Jahrgang 1972, Ausbildung zum Krankenpfleger 1995-1998 am Ev. KH Göttingen-Weende. Danach auf diversen Normalpflege- und Intensivstationen tätig, 2008-2011 Weiterbildung zur Fachkraft für Anästhesie- und Intensivpflege an der UMG Göttingen, 2014 - 2016 Weiterbildung zur Fachkraft für Hygiene in der Pflege am BSG Braunschweig, seit 8/2014 in der Zentralabteilung Krankenhaushygiene und Infektiologie der UMG Göttingen tätig.

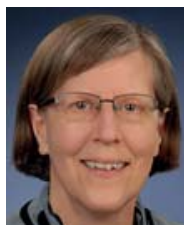

\section{Dr. med. Renate Rettkowski}

Dr. med. Renate Rettkowski ist Mitarbeiterin der Zentralabteilung Krankenhaushygiene und Infektiologie der Universitätsmedizin Göttingen.
Jens Jakisch

Universitätsmedizin Göttingen

Zentralabteilung Krankenhaushygiene

und Infektiologie

Robert-Koch-Str. 40

37075 Göttingen

E-Mail: jens.jakisch@med.uni-goettingen.de

\section{Literatur}

[1] Empfehlung der Kommission für Krankenhaushygiene und Infektionsprävention (KRINKO) beim Robert Koch-Institut. Prävention der nosokomialen beatmungsassoziierten Pneumonie (2013). Bundesgesundheitsbl 2013; 56: 1578 - 1590

[2] Klompas M, Branso R, Eichenwald EC et al. Strategies to Prevent Ventilator-Associated Pneumonia in Acute Care Hospitals: 2014 Update. Infection Control \& Hospital Epidemiology 2014; 35: 915-936

[3] Bundesanstalt für Arbeitsschutz und Arbeitsmedizin (BAUA). Technische Regel für Biologische Arbeitsstoffe 250 GMBI 27.03 2014; 10/11: 206 Änderung: GMBI. Nr. 25 vom 22.5.2014, S. 535; 2. Änderung: GMBI. Nr. 29 vom 21.7.2015, S. 577

\section{Bibliografie}

DOI http://dx.doi.org/10.1055/s-0043-102858

Krankenhaushygiene up2date 2017; 12: 9-13

(c) Georg Thieme Verlag KG Stuttgart · New York ISSN 1862-5797 